\title{
Open issues of interface computation at complementary time and length scales
}

\author{
H. Emmerich ${ }^{\mathrm{a}}$ \\ Materials and Process Simulation, University of Bayreuth, Germany
}

Received: 4 July 2011

Published online: 18 October 2011 - (C) Società Italiana di Fisica / Springer-Verlag 2011

\begin{abstract}
Understanding the dynamics of evolving interfaces is of great interest in most diverse scientific fields as medicine (e.g., the interfaces surrounding growing tumors), chemical engineering (e.g., the interfaces of combustion fronts), physics (e.g., the interfaces seperating different fluids in multiphase flow systems), and materials science (e.g., corroding surfaces of household materials). All those different interfacial systems have in common that their dynamics is usually nonlinear and characterised by complex interfacial properties such as fluctuations, nucleation, aggregation, mass and charge transport. Up to date, there is no single model and corresponding computational approach which could predict all of these properties. One important reason for this, which constitutes at the same time one of the main challenges in modeling and simulating nonlinear interface dynamics, is that those properties are distinguished by multiple length
\end{abstract} and time scales.

Despite this large challenge the past decade has witnessed significant advances in the computational treatment of nonlinear interface dynamics, see, e.g., [1], driven by the steadily increasing economic and technological importance of systems involving such complex evolving interfaces. Consider, for example, the field of materials science: Materials have become an ever more important factor in most advanced technologies. This comes along with a constant quest for new materials, improved functional performance and decreased development costs. In this context, nanoscale assemblies with highly ordered nanoscale building blocks such as quantum dots and quantum wires have shown remarkable optical, electronic, magnetic and mechanical properties that have a wide range of applications. However, such nanoscale building blocks must be fabricated on a surface or through an interface. These processes are usually far from equilibrium. And essentially, as sizes decrease, interfacial properties become more and more dominant.

This demonstrates the steadily growing importance of systematic model and computer simulation methods development adressing the above multiscale problem of nonlinear interface dynamics ranging from the quantum-mechanical to the continuum scale.

Indeed, materials science simulation methods have matured to this point: There has been a growing impact on materials development by modeling and simulation at all relevant length scales (quantum-mechanical, atomic, mesoscopic, continuum) [2-4]. Dedicated methods have been designed for each length scale, as well as methods to address scale-bridging phenomena (multiscale modeling [5]). The success of these methods can be seen in the fact that gradually the emphasis in materials modeling shifted from explaining experimentally observed properties of materials to predicting not yet measured properties.

However, as outlined in [1], such multiscale approaches are by no means standardized yet. They evolve with the given technological demands. More precisely, their development is an ongoing scientific field driven by the collaborative advances of chemists, physicists, mathematicians and computer scientists addressed by the engineers again and again for new specific problems of industrial impact with additional challenges for modeling and simulation. The first provide physically and chemically motivated model derivations, which need to be programmed as efficiently as possible. As for the latter, advanced numerical schemes developed in the related mathematical and computer science departments play a crucial role. Moreover, such models need to be verified either by comparison to experiments or to other model approaches or even to limits where they can by analyzed based on tools from analytical mathematics. The latter analytical mathematics also provides additional methods for model derivation which can be combined with the already mentioned approaches from physics and chemistry to develop enhanced models. For recent advances in nonlinear interface dynamics computation based on such a multidisciplinary approach see [1].

\footnotetext{
${ }^{a}$ e-mail: heike.emmerich@uni-bayreuth.de
} 
This focus point is devoted to selected open issues of interface computation at complementary time and length scales. The contributions therein can be grouped in three classes: i) First there are those contributions which aim at the development of more quantitative simulation models adressing such important interfacial properties as interfacial anisotropy and topological singularities. These are essentially the articles by J. Casademunt on viscous fingering and M. Fleck et al. on phase-field modeling with highly anisotropic interfacial energy. ii) The second class of contributions is concerned with the further development of models whereby the comparison with experiments and analyses, respectively, is thoroughly taken into account and even reassessed to propose more rigorous comparative studies to validate further model development. The contributions falling in this class are the articles by J. Kundin et al., R. Luschtinetz et al., as well as by D. Pilipenko et al. iii) Finally, two contributions of a third class deal with taking the capacity of model approaches to bridge involved time and lenght scales further, namely those of M. Friak et al. and $\mathrm{H}$. Emmerich and L. Granasy.

I hope that these selected contributions constitute illustrative examples of the quality of the technical difficulties imposed by the field and, at the same time, can provide a stimulus to push its, yet demanding, open challenges.

\section{References}

1. H. Emmerich, S. Gemming (Editors), Eur. Phys. J. ST 177, 1 (2009).

2. D. Raabe (Editor), Computational Materials Science - The Simulation of Materials Microstructures and Properties (WileyVCH, 1998) p. 402.

3. J.M. Rickman, R. LeSar, Annu. Rev. Mater. Res. 32, 195 (2002).

4. S. Yip (Editor), Handbook of Materials Modeling (Springer, 2005) p. 2965.

5. Z.X. Guo (Editor), Multiscale Materials Modeling - Fundamentals and Applications (Woodhead Publishing, 2007$)$ p. 350. 